\title{
Metformin attenuates blood-brain barrier disruption in mice following middle cerebral artery occlusion
}

\author{
Yanqun Liu', Guanghui Tang ${ }^{2}$, Yaning Li' ${ }^{2}$, Yang Wang ${ }^{1}$, Xiaoyan Chen², Xiang Gu' ${ }^{2}$ Zhijun Zhang ${ }^{2}$,
} Yongting Wang ${ }^{2,3^{*}}$ and Guo-Yuan Yang ${ }^{1,2,3^{*}}$

\begin{abstract}
Background: Metformin, a widely used hypoglycemic drug, reduces stroke incidence and alleviates chronic inflammation in clinical trials. However, the effect of metformin in ischemic stroke is unclear. Here, we investigated the effect of metformin on ischemic stroke in mice and further explored the possible underlying mechanisms.

Methods: Ninety-eight adult male CD-1 mice underwent 90-minute transient middle cerebral artery occlusion (tMCAO). Metformin (200 mg/kg) was administrated for up to 14 days. Neurobehavioral outcomes, brain infarct volume, inflammatory factors, blood-brain barrier (BBB) permeability and AMPK signaling pathways were evaluated following tMCAO. Oxygen glucose deprivation was performed on bEND.3 cells to explore the mechanisms of metformin in inhibiting inflammatory signaling pathways.

Results: Infarct volume was reduced in metformin-treated mice compared to the control group following tMCAO $(P<0.05)$. Neurobehavioral outcomes were greatly improved in metformin-treated mice $(P<0.05)$. $\mathrm{MPO}^{+}$cells, $\mathrm{Gr} 1^{+}$ cells, MPO activity and BBB permeability were decreased after metformin administration $(P<0.05)$. In addition, metformin activated AMPK phosphorylation, inhibited NF-KB activation, down-regulated cytokine (IL-1 $\beta$, IL-6, TNF-a) and ICAM-1 expression following TMCAO $(P<0.05)$. Furthermore, metformin activated AMPK signaling pathway and alleviated oxygen-glucose deprivation-induced ICAM-1 expression in bEND.3 cells $(P<0.05)$. Compound $C$, a selective AMPK inhibitor, eliminated this promotional effect.

Conclusions: Metformin down-regulated ICAM-1 in an AMPK-dependent manner, which could effectively prevent ischemia-induced brain injury by alleviating neutrophil infiltration, suggesting that metformin is a promising therapeutic agent in stroke therapy.
\end{abstract}

Keywords: Blood-brain barrier, ICAM-1, Inflammation, Ischemic stroke, Metformin

\section{Background}

Ischemic stroke is the second leading cause of death worldwide [1]. Due to its high disability, it is also a big burden on our society. So far, the only Food and Drugs Administration (FDA) approved drug for the treatment of ischemic stroke is rtPA, which improves clinical outcomes if administrated within 4.5 hours after the stroke

\footnotetext{
* Correspondence: yongting.wang@gmail.com; gyyang0626@gmail.com ${ }^{2}$ Neuroscience and Neuroengineering Research Center, Med-X Research Institute and School of Biomedical Engineering, Shanghai Jiao Tong University, Shanghai 200030, China

'Department of Neurology, Ruijin Hospital, School of Medicine, Shanghai Jiao Tong University, Shanghai 200025, China

Full list of author information is available at the end of the article
}

onset. However, due to its narrow therapeutic window, less than $5 \%$ of patients benefit from it [2]. Therefore, developing effective drugs to treat ischemic stroke is an important task.

Metformin is a drug widely prescribed for the treatment of type 2 diabetes and other metabolic syndromes since 1960s [3]. Through activating AMP-activated kinase (AMPK), metformin inhibits hepatic glucose production and increases peripheral glucose utilization, which effectively controls blood glucose level [4]. However, its ability is not limited to lowering glucose. The benefits of metformin have been demonstrated in clinical trials. Metformin reduces stroke incidence and diabetes related 
death [5]. Metformin also reduces intercellular adhesion molecule-1 (ICAM-1) and vascular cell adhesion molecule1 (VCAM-1) levels in plasma and alleviates chronic inflammation in patients [6]. Nevertheless, these effects were independent of its glycemic management properties, suggesting that metformin may have other functions through mechanisms other than glucose reduction. AMPK is a trimetric enzyme comprising a catalytic $\alpha$-subunit and regulatory $\beta$ - and $\gamma$-subunits [7]. An alteration in AMP/ATP ratio activates AMPK and promotes AMPK phosphorylation at a threonine residue (Thr-172) [3]. A series of pathological conditions such as glucose deprivation, ischemia, starvation and oxidative stress increase AMPK activity [8]. Agents such as resveratrol and adiponectin can also activate AMPK. AMPK activation is a protective reaction that occurs after injury. AMPK activated by metformin showed to reduce endothelial cell apoptosis and diminish cardiomyocyte death $[9,10]$. It has been demonstrated that increasing AMPK activity in neurons protects neurons from various injuries [11]. In addition, metformin reduced TNF- $\alpha$-induced inflammation via activation of AMPK in vascular endothelial cells (ECs) [12]. It has been indicated that AMPK activation in cells in the immune system promotes the switch from a pro-inflammatory to an anti-inflammatory phenotype [13]. Thus, metformin could be a promising anti-inflammatory agent.

ICAM-1 is expressed on many cell types including ECs and lymphocytes [14]. ICAM-1 is expressed constitutively on ECs at low level and its expression is significantly increased in hypoxic condition [15]. ICAM-1 expressed on ECs facilitates neutrophil adhesion and tissue infiltration, which play critical roles in the progress of ischemic stroke $[16,17]$. Infiltrated leukocytes induce a secondary injury after reperfusion by producing detrimental substances that damage brain cells and disrupt the blood-brain barrier (BBB) $[18,19]$. BBB disruption after ischemia increases brain edema and exacerbates ischemic injury [14]. Since ICAM-1 plays a vital role in neutrophil infiltration and cerebral injury after reperfusion, it is a promising target in the treatment of ischemic stroke.

Studies have illustrated that metformin provides cardioprotection against myocardial infarction [4]. However, the function of metformin in inflammation after ischemic stroke is unknown. In our research, we explored whether metformin could reduce ischemic brain injury using a mouse transient middle cerebral artery occlusion (tMCAO) model, and attempted to define the underlying mechanism of metformin.

\section{Methods}

\section{Experimental design}

Animal protocol was approved by the Institutional Animal Care and Use Committee of Shanghai Jiao Tong University, Shanghai, China. Ninety-eight adult male CD1 mice were divided into two groups that either underwent metformin or saline treatment. At 1 and 3 days after tMCAO, mice were sacrificed and samples were collected for further study. Metformin (Sigma, St. Louis, MO, USA) was dissolved in sterile saline at a concentration of $30 \mathrm{mg} / \mathrm{ml}$ and $200 \mathrm{mg} / \mathrm{kg}$ was administered intra-peritoneally immediately after reperfusion and then administered daily until the animals were sacrificed. An equal volume of saline was used for the control group. The dose was chosen according to a previous study [20]. The whole experimental design and the number of animal used are displayed in Figure 1.

\section{Transient middle cerebral artery occlusion (tMCAO)} in mice

tMCAO was carried out as previously described [21]. Adult CD1 mice weighing $30 \pm 5$ grams were anesthetized with ketamine/xylazine $(100 \mathrm{mg} / 10 \mathrm{mg} / \mathrm{kg}$; Sigma, St. Louis, MO, USA) through intra-peritoneal injection. After isolation of the left common carotid artery, the external carotid artery (ECA) and the internal carotid artery (ICA), a silicone-coated 6-0 suture (Covidien, Mansfield, MA, USA) was gently inserted into the ICA and stopped at the opening of the middle cerebral artery (MCA). Successful occlusion was ascertained by a decrease of surface cerebral blood flow to $10 \%$ of baseline using a laser Doppler flowmetry (Moor Instruments, Devon, UK). Reperfusion was performed 90 minutes after tMCAO with suture withdrawal. Sham-operated mice underwent the same procedure except for the insertion of the suture into the ICA. The mortality in our study was less than $5 \%$.

\section{Infarct volume measurement}

Infarct volume was measured using cresyl violet (Sigma, St. Louis, MO, USA) staining as previously described [22]. The ischemic area of each section was depicted by image

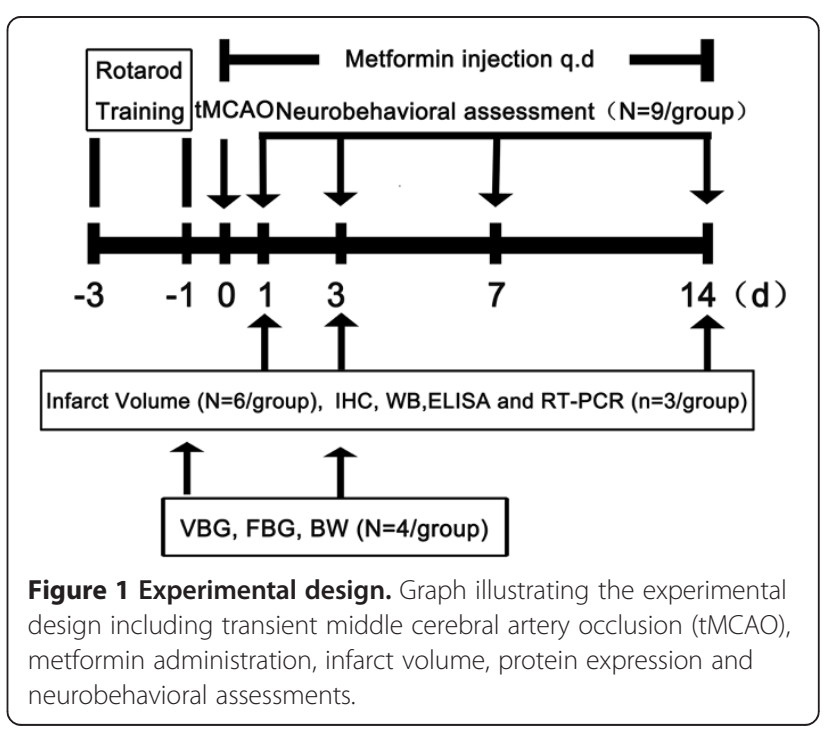


analysis software (Image J, NIH, MD, USA). Infarct volume was calculated as described in our previous study [23].

\section{Neurobehavioral assessments}

Neurobehavioral assessments were conducted by an experimenter who was blind to the treatment conditions. The rotarod test was used to evaluate the motor and balance functions of the mice. The mice were trained to stay on an accelerating rotating cylinder for 3 days before $\mathrm{tMCAO}$, and time remained on the rotating rod was recorded before surgery and at 1, 3, 7 and 14 days after surgery. The velocity was increased slowly from 4 to $40 \mathrm{rpm}$ within 2 minutes. For each test, every animal was tested three times, and the average time maintained on the rod was recorded. For neurological function assessment, a modified Neurological Severity Scores (mNSS) ranging from 0 to 14 score was adopted, which included raising the mouse by the tail (0 to 3 ), walking on the floor ( 0 to 3 ), beam balance tests ( 0 to 6 ), and the relaxes absence (0 to 2$)$ [22].

\section{Immunostaining}

Double staining: ZO-1/CD31, occludin/CD31 or claudin$5 /$ CD31 double staining was conducted as previously described [23]. Briefly, brain sections were blocked with $10 \%$ FBS for 1 hour and then incubated with ZO-1 (1:100 dilution, Invitrogen, Carlsbad, CA, USA) and CD31 (1:200 dilution, R\&D Systems, Minneapolis, MN, USA); occludin (1:100 dilution, Invitrogen, Carlsbad, CA, USA) and CD31; claudin-5 (1:100 dilution, Invitrogen, Carlsbad, CA, USA) and $\mathrm{CD} 31$ at $4^{\circ} \mathrm{C}$ overnight. After washing, brain sections were incubated with the appropriate second antibodies for 1 hour. Brain sections were examined using a confocal microscope (Leica, Solms, Germany) and photographs were taken for further analysis.

DAB staining: for myeloperoxidase (MPO), ICAM-1 and Gr1 (Ly 6G) immunostaining, brain sections were incubated in $0.3 \% \mathrm{H}_{2} \mathrm{O}_{2}$ in methanol for 30 minutes. After blocking with FBS, the primary anti-body MPO (1:300 dilution, R\&D Systems, Minneapolis, MN, USA) and ICAM-1 (1:200 dilution, R\&D Systems, Minneapolis, MN, USA), Gr1 (1:100 dilution, Millipore, Darmstadt, Germany) were incubated overnight at $4^{\circ} \mathrm{C}$. Sections were incubated with biotinylated-conjugated secondary antibody (Vector Laboratories, Burlingame, CA, USA) and then incubated with Vectastain $A B C$ Reagent. The reaction product was visualized using a DAB substrate (Vector Laboratories, Burlingame, CA, USA). Eight interested fields in each ipsilateral hemisphere, including the perifocal region in both cortex and striatum, were photographed in each section and five consecutive sections spaced at $200 \mu \mathrm{m}$ were counted in each mouse. $\mathrm{MPO}^{+}$and $\mathrm{Grl}^{+}$(Ly 6G) cells were counted in each field by a person blinded to the treatment group. IgG leakage was examined as previously reported and the procedure was similar to MPO staining except for the primary antibody incubating process [23]. Four areas of ischemic penumbra from each slide were photographed. And mean optical density was measured using Image-Pro Plus software (Media Cybernetics, Bethesda, MD, USA).

\section{Western blot analysis}

Samples were lysed in radioimmunoprecipitation assay (RIPA) (Millipore, Bedford, MA, USA) supplemented with $1 \mathrm{mmol} / \mathrm{L}$ PMSF (Thermo, Waltham, MA, USA), cocktail (Thermo, Waltham, MA, USA) and phosphatase inhibitor (Thermo, Waltham, MA, USA). For Western blot analyses, samples containing the same amount of proteins were loaded onto the resolving gel (Promoton, Shanghai, China) for electrophoresis after denaturing. Proteins were transferred onto a nitrocellulose membrane (Whatman, Piscataway, NJ, USA). After being blocked with 5\% non-fat milk, the membrane was incubated with primary antibodies at the following dilution MPO (1:500), ICAM-1 (1:2,000), ZO-1 (1:500), occludin (1:500), claudin-5 (1:500), p-AMPK (1:1,000, Cell Signaling Technology, Beverly, MA, USA), AMPK (1:1,000, Cell Signaling Technology, Beverly, MA, USA), p-NF-кB (1:1,000, Cell Signaling Technology, Beverly, MA, USA), NF-кB (1:1,000, Cell Signaling Technology, Beverly, MA, USA), $\beta$-actin $(1: 1,000$, Santa Cruz Technology, Santa Cruz, CA, USA) at $4^{\circ} \mathrm{C}$ overnight, respectively. After washing, the membrane was incubated with the appropriate horseradish peroxidase (HRP)-conjugated secondary antibody for 1 hour and then reacted with enhanced chemiluminescence substrate (Pierce, Rockford, IL, USA). The results were recorded by Quantity One image software (Bio-Rad, Hercules, CA, USA) and relative intensity was calculated using Gel-Pro Analyzer software (Media Cybernetics, Bethesda, MD, USA).

\section{MPO activity assay}

MPO activity assay was performed as described previously [23]. In brief, brain protein $(10 \mu \mathrm{L})$ from ipsilateral hemisphere was added to $180 \mu \mathrm{L}$ of work solution, which contained $2 \mathrm{mmol} / \mathrm{L}$ O-dianisidin-dihydrochloride (Sigma) dissolved in $180 \mu \mathrm{l}$ of $50 \mathrm{mmol} / \mathrm{L}$ potassium phosphate buffer ( $\mathrm{pH}=6)$. Before measurement, $10 \mu \mathrm{L}$ of $100 \mathrm{mmol} / \mathrm{L}$ $\mathrm{H}_{2} \mathrm{O}_{2}$ was added. Changes in absorbance at $460 \mathrm{~nm}$ over 10 minutes were measured. MPO activity was expressed as $\mathrm{U} / \mathrm{mg}$ tissue, and $1 \mathrm{U}$ of MPO activity represents the amount of enzyme degrading $1 \mu \mathrm{mol} \mathrm{H}_{2} \mathrm{O}_{2}$ per minute at $25^{\circ} \mathrm{C}$.

\section{Real-Time PCR}

Total RNA from the ischemic hemisphere was extracted using TRIzol reagent (Invitrogen, Carlsbad, CA, USA) and dissolved in $60 \mu \mathrm{L}$ RNA free water according to the 
manufacturer's instructions. A universal 2-step RT-PCR cycling condition was used: $95^{\circ} \mathrm{C}$ for 30 seconds followed by 40 cycles of $95^{\circ} \mathrm{C}$ for 5 seconds and $60^{\circ} \mathrm{C}$ for $30 \mathrm{sec}-$ onds. mRNA levels were normalized to the endogenous control, GAPDH expression, and were calculated using fold change relative to the saline control group [23].

\section{Enzyme Linked Immunosorbent Assay (ELISA) analysis}

Protein levels of IL-1 $\beta$, IL-6, and TNF- $\alpha$ were quantified using an ELISA kit (R\&D systems, Minneapolis, MN, USA) according to the manufacturer's instruction. Absorbance at $450 \mathrm{~nm}$ was recorded and the concentration of the target protein was read according to the standard curve. Result was expressed as pg/mg protein.

\section{Evans blue extravasation}

Evans blue extravasation was measured as previously described. In brief, 3 days after tMCAO, $4 \mathrm{ml} / \mathrm{kg}$ of $2 \%$ Evans blue (Sigma, St. Louis, MO, USA) in saline was administered intraperitoneally. After 2 hours circulation, mice were anesthetized and perfused with saline through the left ventricle until colorless fluid outflowed from the right atrium. Then, ipsilateral and contralateral hemispheres were collected after decapitated. Each hemisphere was weighed rapidly, homogenized in $1 \mathrm{ml}$ of $50 \%$ trichloroacetic acid (wt/vol). After centrifugation (12,000 × $g, 20$ minutes), supernatant was collected and mixed with ethanol (1:3). The concentration of Evans blue was determined by measuring the $610 \mathrm{~nm}$ absorbance and tissue content of Evans blue was quantified from a linear standard curve and expressed in terms of Evans blue ( $\mu \mathrm{g}) /$ tissue $(\mathrm{g})$.

\section{Oxygen glucose deprivation}

bEND.3 was purchased from American Type Culture Collection (ATCC) and cultured in DMEM (Gibco Laboratories, Grand Island, NY, USA) supplemented with $10 \%$ FBS. Ischemia-like conditions in vitro were induced by oxygen glucose deprivation and reperfusion-like conditions in vitro were induced by reoxygenation. After cells reached a $90 \%$ confluence, the medium was replaced with DMEM without glucose. Then, cultures were transferred to an anaerobic chamber infused with a gas mixture containing $5 \% \mathrm{CO}_{2}, 95 \% \mathrm{~N}_{2}$. After incubating for 6 hours, cells were further cultured in DMEM supplemented with $10 \%$ FBS under normal conditions for another 24 hours with or without metformin. p-AMPK analysis in bEND.3 cells was determined 1 hour, 4 hours, and 24 hours after 6 hours oxygen glucose deprivation (OGD). The dose used was $10 \mathrm{mM}$, which was chosen according to previous reports [20]. Compound C (Sigma, St. Louis, MO, USA), a selective AMPK inhibitor was added to the medium at a final concentration of $10 \mu \mathrm{mol} / \mathrm{L}$ before OGD treatment and maintained throughout the whole experiment [24]. An equal volume of PBS was used in the control group.

\section{Statistical analysis}

Results were presented as mean \pm SD. Statistical analysis was evaluated by Prism 4 software (GraphPad Software, San Diego, CA, USA). For comparison between the two groups, statistical significance was determined through a Student's t test. For comparison among multiple groups, statistical significance was evaluated using one-way ANOVA followed by a Student-Newman-Keuls test. A probability value of $P<0.05$ was considered statistically significant.

\section{Results}

\section{Metformin reduced infarct volume and improved} neurobehavioral outcomes

We found that after metformin treatment, infarct volume was reduced at 1 and 3 days after tMCAO (Figure 2A-B). To further explore the function of metformin, we used mNSS to examine the motor, balance and reflex functions of mice after tMCAO. We showed that mice had significantly lower scores after 3 days in the metformintreated group after tMCAO for at least 14 days (Figure 2C). A similar result was obtained from the rotarod test (Figure 2D). To investigate whether metformin influenced glucose levels after tMCAO, fasting blood-glucose (FBG) was tested before, 3 days and 14 days after tMCAO, indicating metformin did not influence FBG level, blood gas or body weight after both 3 (Table 1) days and 14 days treatment (Data were shown in Additional file 1: Table S1)

\section{Metformin alleviated neutrophil infiltration and IL-1 $\beta$, IL-6, TNF- $a$ expression}

To investigate the effect of metformin on neutrophil infiltration in the acute phase of cerebral ischemia, we performed 3,3'-diaminobenzidine (DAB) staining to detect $\mathrm{MPO}^{+}$cells. Results showed $\mathrm{MPO}^{+}$cells were almost undetectable in the sham group, and there was a decrease in $\mathrm{MPO}^{+}$cells at 1 and 3 days after tMCAO in the metformin-treated group compared to the control group (Figure 3D). Western blot analysis indicated that MPO were reduced in metformin-treated mice $(P<0.01$, Figure 3E). MPO activity is an indicator of inflammation and could be used to evaluate neutrophil accumulation [23]. MPO activity was attenuated in the metformintreated group compared to the control group $(P<0.05$, Figure $3 C$ ). In addition, we used another neutrophil marker Gr1 (Ly 6G) to evaluate neutrophil infiltration after cerebral ischemia. Results showed that metformin reduced Gr1 positive cells effectively at 1 day and 3 days after tMCAO (Additional file 2: Figure S1, $P<0.01$ ). We further used RT-PCR and ELISA to evaluate changes in inflammation-related cytokine expression in mRNA and protein levels. Metformin reduced IL- $\beta$, IL-6, TNF- $\alpha$ mRNA at 1 day after tMCAO. Although there was a downward tendency in IL-6 mRNA, only changes in IL- $\beta$, 

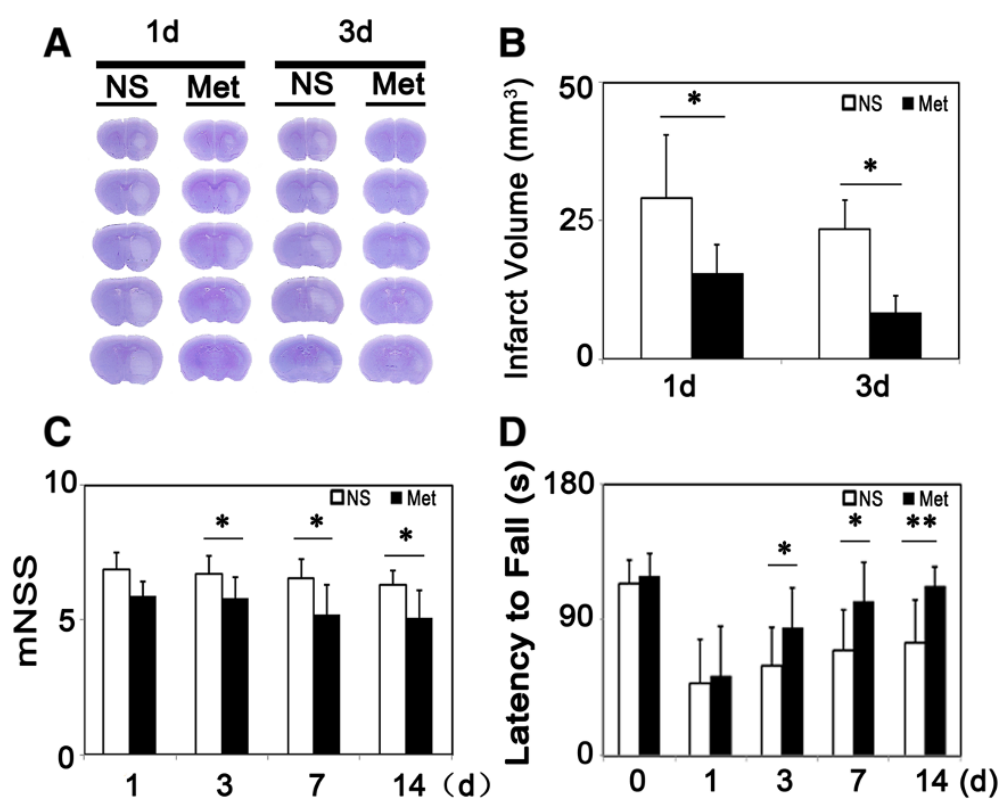

Figure 2 Metformin reduced infarct volume and improved neurobehavioral outcomes following transient middle cerebral artery (tMCAO) in mice. (A) Photographs show a series of coronal sections with cresyl violet staining following tMCAO in metformin- and saline-treated mice. (B) Bar graph shows a quantification of the infarct volume from (A). $n=6$ per group. The changes in neurological scores (C) and the rotarod test (D) at 1, 3,7 and 14 days following tMCAO in metformin- and saline-treated mice. $n=9$ per group, data are mean $\pm S D,{ }^{*} P<0.05$, metformin versus control group, ${ }^{*} P<0.01$, metformin versus control group.

and TNF- $\alpha$ were statistically significant in the metformin group compared to the control group at 3 days after tMCAO (Figure 3A). There was a decrease of IL- $\beta$, IL-6, TNF- $\alpha$ expression at 3 days after $\mathrm{TMCAO}$ in protein level (Figure 3B, $P<0.01$ ).

Table 1 Metformin did not influence blood gas, glucose level and body weight in transient middle cerebral artery occlusion (tMCAO) mice

\begin{tabular}{llllll}
\hline & \multicolumn{2}{l}{ Before tMCAO } & & \multicolumn{2}{l}{ After tMCAO } \\
\cline { 2 - 3 } \cline { 6 - 6 } \cline { 5 - 6 } & NS & Met & & Net \\
\hline $\mathrm{pH}$ & $7.35 \pm 0.05$ & $7.34 \pm 0.03$ & & $7.34 \pm 0.07$ & $7.35 \pm 0.03$ \\
$\mathrm{PCO}_{2}(\mathrm{mmHg})$ & $38 \pm 5$ & $33 \pm 3$ & & $30 \pm 7$ & $30 \pm 5$ \\
$\mathrm{PO}_{2}(\mathrm{mmHg})$ & $62 \pm 4$ & $60 \pm 7$ & $64 \pm 2$ & $66 \pm 12$ \\
$\mathrm{SO}_{2}(\%)$ & $91 \pm 8 \%$ & $87 \pm 10 \%$ & $91 \pm 2 \%$ & $91 \pm 5 \%$ \\
$\mathrm{Na}(\mathrm{mmol} / \mathrm{L})$ & $155 \pm 2$ & $153 \pm 2$ & & $154 \pm 3$ & $152 \pm 2$ \\
$\mathrm{~K}(\mathrm{mmol} / \mathrm{L})$ & $3.2 \pm 0.1$ & $3.1 \pm 0.4$ & & $3.5 \pm 0.3$ & $3.9 \pm 0.2$ \\
$\mathrm{iCa}(\mathrm{mmol} / \mathrm{L})$ & $1.37 \pm 0.02$ & $1.34 \pm 0.06$ & & $1.22 \pm 0.04$ & $1.26 \pm 0.04$ \\
$\mathrm{Glu}(\mathrm{mg} / \mathrm{dL})$ & $132 \pm 20$ & $127 \pm 33$ & & $123 \pm 16$ & $129 \pm 24$ \\
$\mathrm{Hct}(\% \mathrm{PCV})$ & $37 \pm 1 \%$ & $35 \pm 1 \%$ & & $34 \pm 6 \%$ & $35 \pm 2 \%$ \\
$\mathrm{Hb}(\mathrm{g} / \mathrm{dL})$ & $12.4 \pm 0.4$ & $11.9 \pm 0.3$ & & $11.4 \pm 2.2$ & $11.8 \pm 0.7$ \\
Body weight $(\mathrm{g})$ & $33 \pm 2$ & $32 \pm 1$ & & $25 \pm 6$ & $28 \pm 4$ \\
\hline
\end{tabular}

Table showed vein blood gas analysis results, glucose levels and body weight at 1 day before $\mathrm{TMCAO}$ and at 3 days after TMCAO in metformin-(Met) and saline(NS)-treated mice ( $n=4$ per group). Data were mean \pm SD.

Legend: Glu, glucose; $\mathrm{Hb}$, hemoglobin; $\mathrm{Hct}$, hematocrit; $\mathrm{PCO}_{2}$, partial pressure of $\mathrm{CO}_{2} ; \mathrm{PO}_{2}$, partial pressure of $\mathrm{O}_{2} ; \mathrm{PCV}$, packed cell volume; $\mathrm{SO}_{2}$, oxygen saturation.

\section{Metformin reduced BBB disruption}

To evaluate endothelial cell permeability after metformin treatment, we conducted occludin/CD31, ZO-1/CD31 and claudin-5/CD31 double staining to observe tight junction distribution in situ at 3 days after tMCAO. Result indicated that occludin and ZO-1 were continuously located on the margin of ECs in sham group, claudin- 5 was continuously located along ECs, and fewer gaps were formed in the metformin-treated group (Figure 4A). Gap formation and rearrangement were used to evaluate tight junction disruption after injury. To evaluate tight junction rearrangement, Western blot was adopted and we found that metformin-treated mice demonstrated occludin, ZO1 and claudin- 5 hyper-expression (Figure 4B). In addition, we performed IgG immunostaining and Evans blue extravasation to evaluate endothelial permeability and found that there was significantly reduced IgG and Evans blue leakage at 3 days after tMCAO in metformin-treated mice (Figure 4C-D).

\section{Metformin down-regulated ICAM-1 expression via AMPK signaling pathway}

To assess the phosphorylation status of AMPK at threonine residue, Western blot was used. We demonstrated that ischemia-reperfusion increased AMPK phosphorylation and this induction was increased after metformin treatment (Figure 5A). To further explore mechanisms of metformin in neuroprotection, we analyzed ICAM-1 expression after 


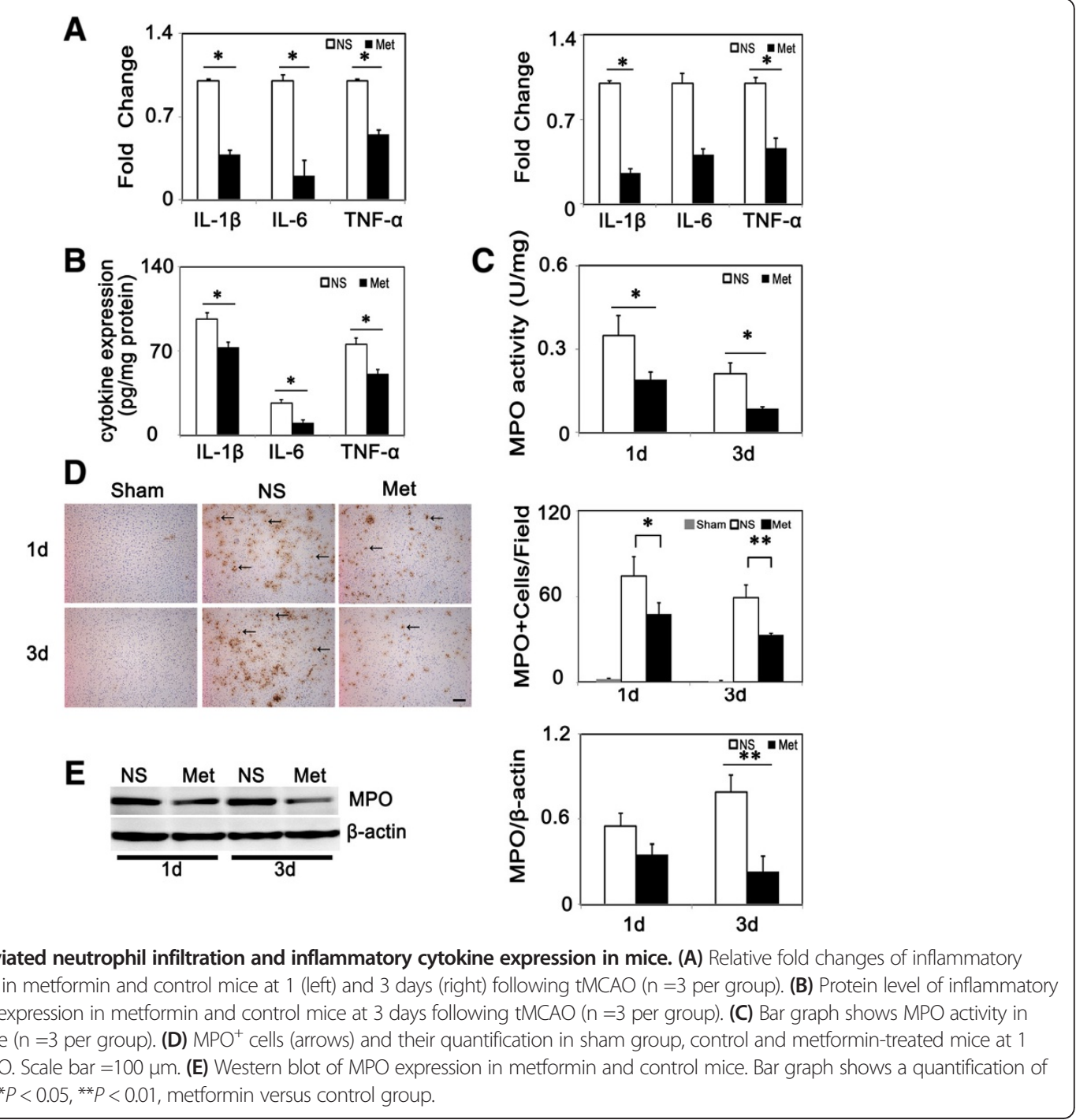

tMCAO. ICAM-1 was expressed constituently at low level in ECs, and after cerebral ischemia its expression was elevated hugely (Figure 5D). Furthermore, Western blot and RT-PCR results indicated that ICAM-1 was reduced after metformin-treatment compared to the control group (Figure $5 \mathrm{~B}-\mathrm{C}$ ). We also found that metformin inhibited NF-KB phosphorylation at 1 day and 3 days after $\mathrm{tMCAO}$ (Figure 5E). To determine whether metformin-induced down-regulation of ICAM-1 was AMPK-dependent or not, we analyzed the effect of metformin at a cellular level, using OGD models to mimic in vivo ischemia/reperfusion injury. First, we treated bEND.3 cells with metformin to determine whether metformin could also increase $\mathrm{p}$ AMPK in vitro. We found that there was an increase in $\mathrm{p}-$ AMPK expression at 60 and 120 minutes after metformin treatment: the maximum effect was at 60 minutes in normal conditions (Figure 6A) and while treatment with metformin after 6 hours of OGD, there was also an increase in p-AMPK expression 1 hour after reoxygenation and $\mathrm{p}$-AMPK levels were unregulated for at least 24 hours after reoxygenation (Figure 6B). Second, we examined ICAM-1 expression after 6 hours of OGD and reoxygenation with or without metformin. After OGD/reoxygenation treatment, ICAM-1 expression was up-regulated in mRNA level and metformin inhibited this up-regulation: the inhibitive effects began at 4 hours after reoxygenation and were sustained for at least 24 hours after reoxygenation (Figure 6C). To test whether AMPK signaling was involved, a selective AMPK inhibitor, compound $C$, was used to block the AMPK phosphorylation. First, we found that compound $\mathrm{C}$ reduced metformin-induced AMPK phosphorylation, then, we used RT-PCR and Western blot to assess ICAM-1 expression after treatment. Results indicated that metformin reduced ICAM-1 expression in both 


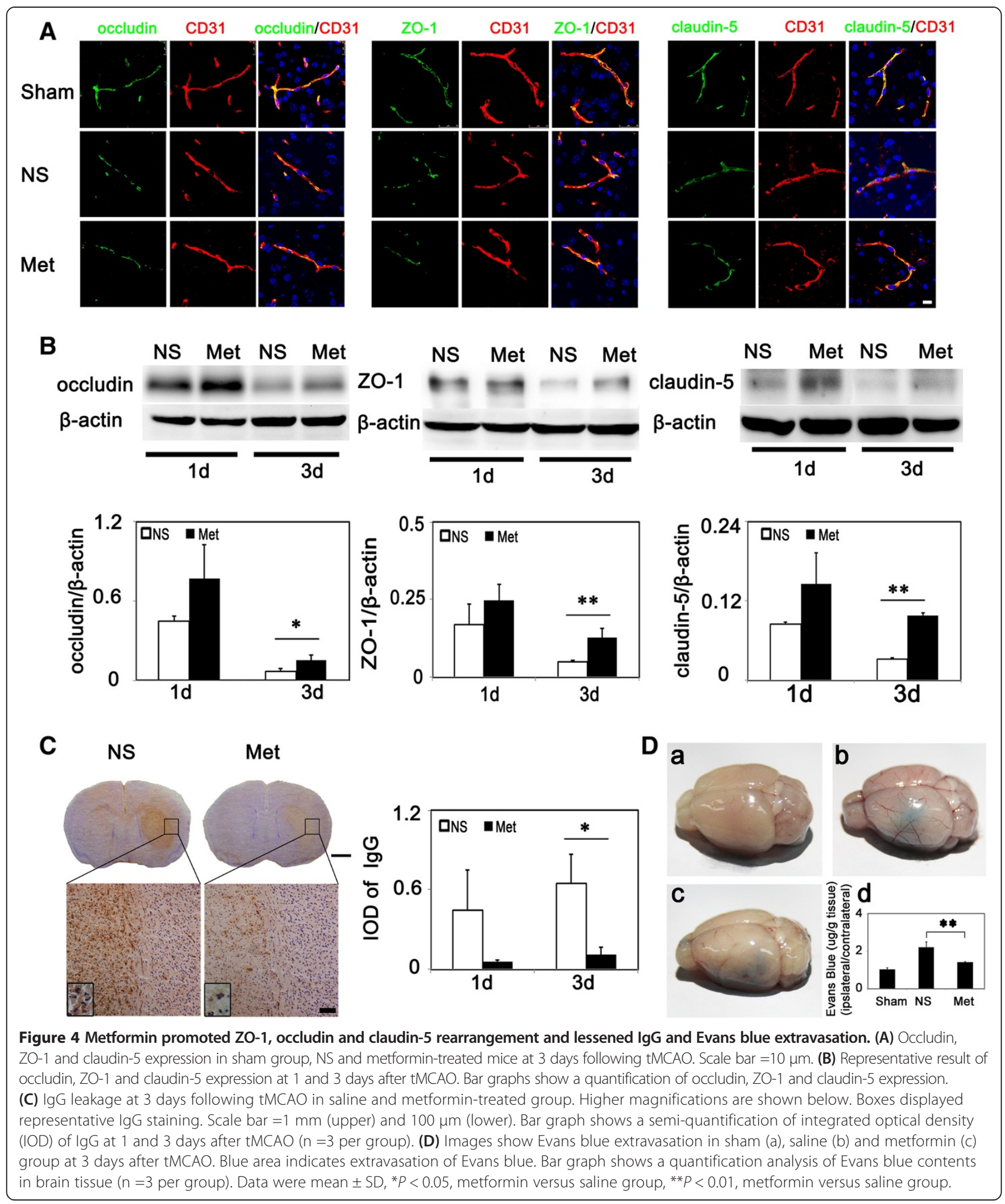

mRNA and protein levels under OGD/reoxygenation conditions (Figure 6D-E). Furthermore, we used Western blot to evaluate the effects of metformin on NF- $\mathrm{kB}$ activation; the result indicated that metformin inhibited NF- $\mathrm{KB}$ phosphorylation and this function was abolished by compound C (Figure 6G). Thus, we concluded that metformin diminished ICAM-1 via an AMPK mediated signaling pathway and AMPK-NF-KB might be involved. 
A

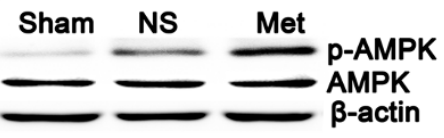

B

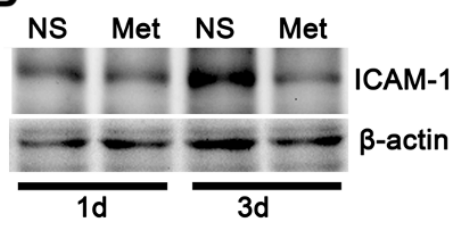

C

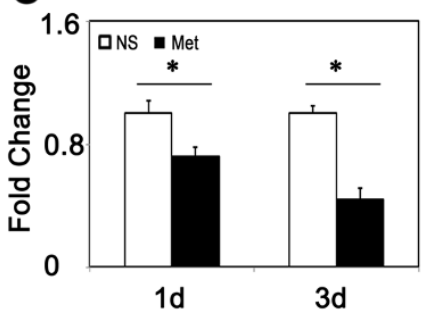

E

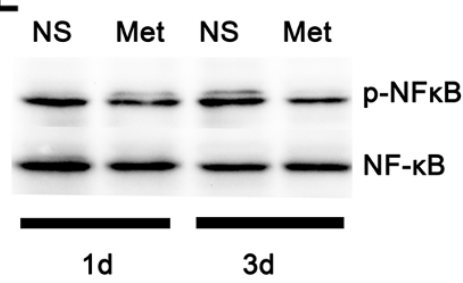

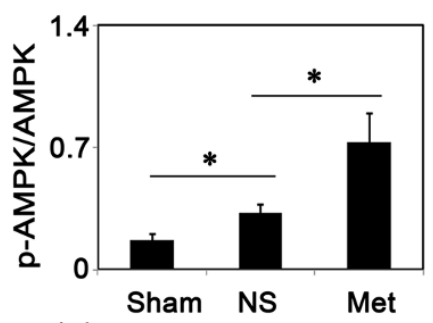

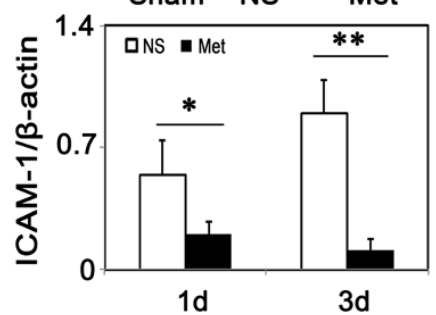

D

$1 \mathrm{~d}$
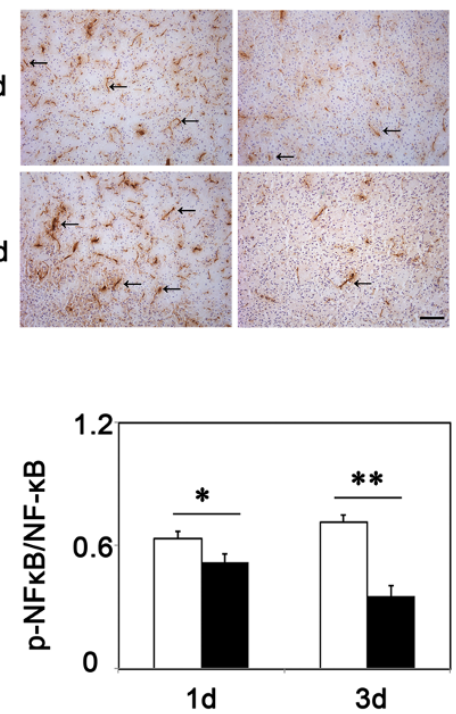

Figure 5 Metformin promoted phosphorylation of AMPK and reduced ICAM-1 expression in transient middle cerebralartery occlusion (tMCAO) mice. (A) p-AMPK and AMPK expression in sham, saline- and metformin-treated groups at 1 day after tMCAO. Bar graph showed a quantification of p-AMPKAMPK ratio ( $\mathrm{n}=3$ /group). (B) ICAM- 1 expression and quantification at 1 and 3 days after tMCAO in metformin and control mice ( $n=3$ per group). (C) Fold change of ICAM-1 expression in mRNA level in metformin and control mice at 1 day and 3 days after tMCAO ( $n=3$ per group). (D) ICAM-1 expression in control and metformin-treated mice at 1 and 3 days after tMCAO. Arrows indicated representative ICAM-1 expression. Scale bar $=100 \mu \mathrm{m}$. (E) p-NF-KB and NF-KB expression in saline- and metformin-treated groups at 1 day and 3 days after tMCAO. Bar graph show a quantification of $\mathrm{p}-\mathrm{NF}-\mathrm{kB} / \mathrm{NF}-\mathrm{kB}$ ratio ( $\mathrm{n}=3$ per group). Data are mean $\pm \mathrm{SD},{ }^{*} P<0.05$, metformin versus control, sham versus control group, ${ }^{*} P<0.01$, metformin versus control group.

\section{Discussion}

In the present study, we demonstrated that metformin protected the brain from ischemic injury through alleviating inflammatory responses in $\mathrm{tMCAO}$ mice, thus improving long-term recovery. Metformin diminished neutrophil infiltration, thereby alleviating endothelial injury and lowering BBB permeability. These effects were potentially mediated via an AMPK-dependent ICAM-1 down-regulation. We also found that inhibiting AMPK activation by compound $\mathrm{C}$ could reverse metformin-induced down-regulation of ICAM-1 in vitro. Thus, we concluded that metformin exerts its protective effect after cerebral ischemia partly through diminished ICAM-1 expression.

Metformin is a glucose-lowering agent, and is one of the first-line drugs recommended to treat type II diabetes mellitus [25]. The UK Prospective Diabetes Study (UKPDS) has revealed that metformin reduced the risk of all-cause mortality and stroke clinically; however, these benefits were independent of its anti-hyperglycemic effects, since metformin reduced glycated hemoglobin $\left(\mathrm{HbA}_{1 \mathrm{c}}\right)$ to the same extent as sulphonylurea and insulin [5]. Metformin decreased myocardial injury in non-diabetic and diabetic 
A

Cont $30 \mathrm{~min} 60 \mathrm{~min} 120 \mathrm{~min}$

P-AMPK

$\longrightarrow$ AMPK

B

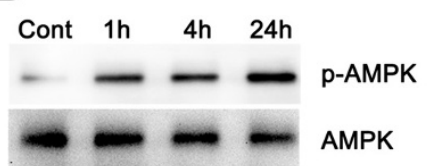

C

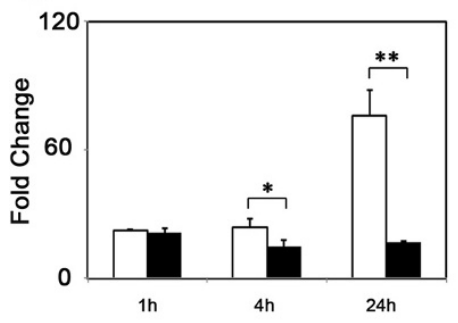

E

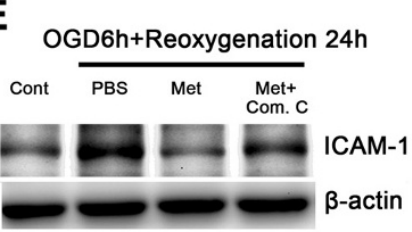

$\mathbf{F}$

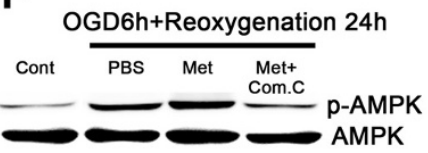

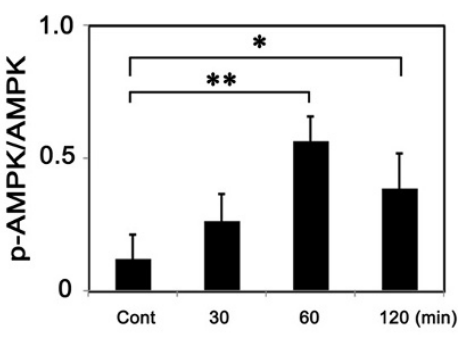

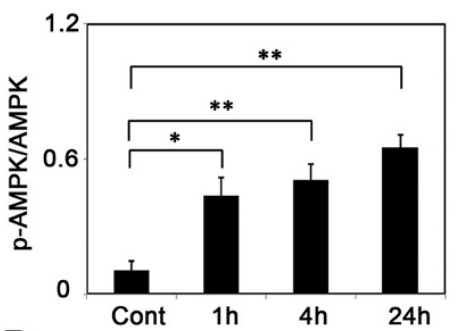

D
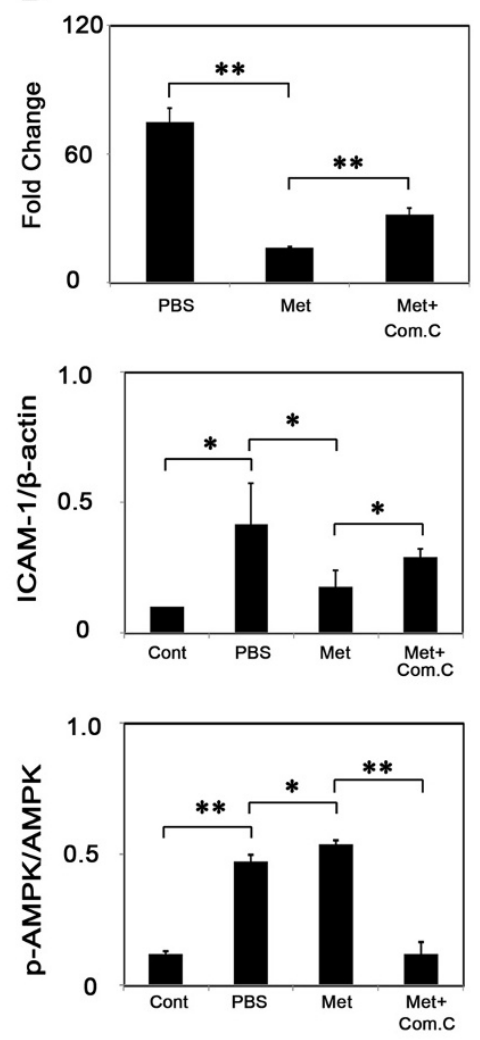

G

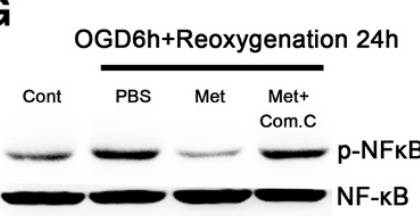

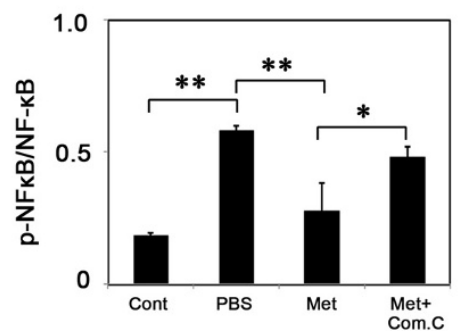

Figure 6 (See legend on next page.) 


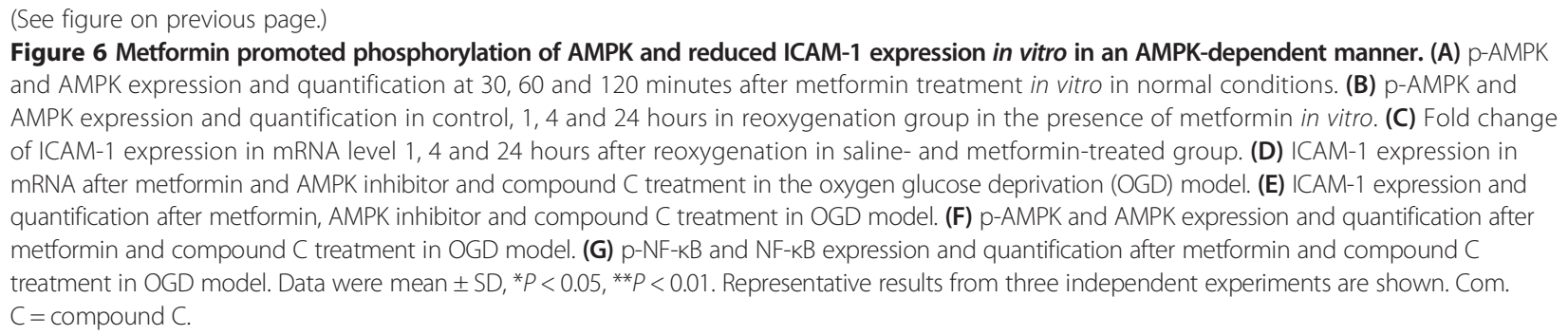

mice [4] and prevented the progression of heart failure in dogs [10]. However, reports regarding neuroprotection of metformin in cerebral ischemia were controversial. Using a 90-minute tMCAO model, McCullough demonstrated that chronic treatment, both pre- and post- (3 weeks), with metformin reduced infarct volume effectively; however, pre-treatment (3 days) enhanced injury in ischemic stroke [26]. Harada et al. showed that using 3-day metformin treatment after 2 hours of tMCAO, metformin effectively reduced infarct volume [27], which was consistent with our result. In addition, $\mathrm{Li}$ has suggested that chronic treatment (14 days in drinking water, $300 \mathrm{mg} / \mathrm{kg}$ ) with metformin in diabetic rats was protective, but acute treatment ( 1 day in drinking water, $300 \mathrm{mg} / \mathrm{kg}$ ) exerted different effects [28]. However, we must note that different models were used. For acute treatment, 3-hour occlusion and 21 hours reperfusion model were used; however, for the chronic treatment, a 90-minute occlusion and 14 days reperfusion model was adopted, and glucose level was normal in chronic treatment rats. Thus, different animal strains and different models may explain the different effects observed from different studies [26]. In our study, using a 90-minute tMCAO model in mice and treatment at the time of reperfusion, we demonstrated that metformin could alleviate ischemic injury and improve neurobehavioral outcomes.

We demonstrated the protection by metformin on ischemic stroke involved, at least in part, AMPK activity [3]. However, McCullough's group found that metformin enhanced ischemic injury in tMCAO animals in a 3-day precondition study through activating AMPK [26]. We believe this result can be due to the period of treatment. Metformin could increase AMPK phosphorylation both in vitro and in vivo $[4,9]$. We detected an induction of AMPK phosphorylation in both bEND. 3 cells and the mouse brain after tMCAO. Notably, conditions that could activate AMPK have been proven to be beneficial in stress, particularly in ischemia. Ischemic precondition, which can activate AMPK due to an increase of the AMP/ATP ratio, is supposed to be protective in ischemic stroke [29]. Adiponectin reduces infarct size in cerebral ischemia and myocardial injury has been shown to be partly through promoting AMPK phosphorylation [30,31]. In addition, AMPK involves pleiotropic pathways that play critical roles in cerebral ischemia. Through suppression of the mTOR signaling pathway, AMPK regulates cell growth and autophagy [7]. Via activation of the Nrf2/ SKN-1 signaling pathway, AMPK increases antioxidant gene expression [32]. By promotion of the eNOS pathway, AMPK reduces endothelial cell apoptosis and improves endothelial functions [9]. Furthermore, emerging evidence shows that AMPK is beneficial to neurons suffering from injuries such as ischemia, starvation, and oxidative damage [11]. Collectively, these results suggest that metforminAMPK signaling pathways exert protective effects in stress conditions and protect the brain from ischemic stroke.

The mechanism by which metformin reduces inflammation after cerebral ischemia is poorly understood. ICAM-1 expression can be regulated by the nuclear transcriptional factor NF- $\mathrm{kB}$ [33]. Recently, the potency of metformin blocking NF- $\mathrm{KB}$ signaling has been illustrated [34]. In addition, metformin decreased TNF- $\alpha$-induced ICAM-1 by inhibiting NF-kB activation in ECs [12]. Therefore, we suppose that metformin reduced ICAM-1 expression via the AMPK-NF- $\kappa B$ pathway. We also detected an inhibition of NF- $\mathrm{kB}$ activation after metformin treatment both in vivo and in vitro. Previous study showed that there was a huge increase of ICAM-1 expression at the time of reperfusion; in our study, metformin was administrated at the time of reperfusion. Increased expression of adhesion cytokine is detrimental during ischemic injury since it increases neutrophil adhesion to ECs and thus promotes their infiltration [16]. Anti-ICAM-1 treatment significantly reduced infarct volume [15]. Coincidently, we demonstrated that metformin decreased ICAM-1 both in vivo and in vitro, and this reduction in ICAM-1 in vivo was accompanied by alleviated neutrophil infiltration and reduced infarct size in tMCAO mice. Furthermore, our study indicated that this effect was possibly mediated by AMPK in a dependent manner. We concluded that metformin conferred resistance to ischemic stroke through decreasing ICAM-1 via the AMPK signaling pathway.

Besides increasing inflammation, neutrophil infiltration also induces EC injury and increases $\mathrm{BBB}$ permeability [35]. BBB disruption exacerbates brain injury after ischemia. Injuries such as ischemia and trauma lead to a disruption and reconstruction of $\mathrm{ZO}-1$ and occludin, and an increase in BBB permeability [23]. Reduction of BBB permeability alleviates cerebral ischemia injury in both 
transient and permanent cerebral ischemia [19,22,23]. Recently, it has been reported that metformin-induced improvement of BBB functions in ECs in vitro is due to activating AMPK activity [24]. In the present study, we demonstrated that after metformin treatment, IgG and Evans blue leakage was significantly reduced and tight junction protein profoundly increased, leading to better outcomes in $\mathrm{TMCAO}$ mice.

\section{Conclusions}

We demonstrated that metformin is beneficial to the treatment of ischemic stroke, which is possible through inhibiting inflammation via AMPK signaling pathways. Since metformin is a widely used drug with few adverse effects, using this long-established drug for a new use may be a promising way to develop an effective therapy for ischemic stroke [36]. Metformin has the potential to be useful in the clinical treatment of ischemic stroke.

\section{Additional files}

Additional file 1: Table S1. Metformin did not influence blood gas, glucose level and body weight 14 days after transient middle cerebral artery occlusion (tMCAO) in mice.

Additional file 2: Figure S1. Metformin reduced neutrophil infiltration in transient middle cerebral artery occlusion (tMCAO) mice. Gr1 (Ly 6G) ${ }^{+}$ cells (arrows) and their quantification in control and metformin treated mice at 1 and 3 days following tMCAO ( $n=3$ /group). Scale bar $=100 \mu \mathrm{m}$. Data are mean $\pm \mathrm{SD},{ }^{*} P<0.01$, metformin versus control group.

\section{Abbreviations}

AMPK: activating AMP-activated kinase; BBB: blood-brain barrier; DAB: 3,3'-diaminobenzidine; ECA: external carotid artery; ECs: endothelial cells; ELISA: Enzyme Linked Immunosorbent Assay; FBG: fasting blood-glucose; ICA: internal carotid artery; ICAM-1: intercellular adhesion molecule-1; IOD: integrated optical density; MCA: middle cerebral artery; mNSS: modified Neurological Severity Scores; MPO: myeloperoxidase; OGD: oxygen-glucose deprivation; tMCAO: transient middle cerebral artery occlusion; VCAM-1: vascular cell adhesion molecule-1.

\section{Competing interests}

The authors declare that they have no competing interests.

\section{Authors' contributions}

YL was involved in research design, experimental performances except animal surgery and neurobehavioral tests, and data analysis as well as drafting the manuscript. GT performed animal surgery. YL and $Y W$ participated in animal behavioral tests. $X C$ and $X G$ gave technical assistant. ZZ discussed the results and edited part of manuscript. YW and GYY are the corresponding authors; they took care of all aspects including research design, data analysis and manuscript preparation. All authors read and approve the final manuscript.

\section{Acknowledgements}

This work was supported by China 973 Program 2011CB504405 (GYY and YW), the National Natural Science Foundation of China, U1232205 (GYY) and 81371305 (YW), Science and Technology Commission of Shanghai Municipality, 13140903500 (GYY) and 13ZR1422600 (ZJZ), Shanghai Jiao Tong University Foundation for technological innovation of major projects 12X190030021 (GYY), and KC Wong Foundation.

\section{Author details}

'Department of Neurology, Ruijin Hospital, School of Medicine, Shanghai Jiao Tong University, Shanghai 200025, China. ${ }^{2}$ Neuroscience and Neuroengineering Research Center, Med-X Research Institute and School of Biomedical Engineering, Shanghai Jiao Tong University, Shanghai 200030, China. Department of Neurology, Med-X Research Institute and School of BME, Ruijin Hospital, School of Medicine, Shanghai Jiao Tong University, 1954 Hua-shan Road, Shanghai 200030, China.

Received: 18 April 2014 Accepted: 30 September 2014

Published online: 15 October 2014

\section{References}

1. Mathers CD, Boerma T, Ma Fat D: Global and regional causes of death Br Med Bull 2009, 92:7-32.

2. Kleindorfer D, Lindsell CJ, Brass L, Koroshetz W, Broderick JP: National US estimates of recombinant tissue plasminogen activator use: ICD-9 codes substantially underestimate. Stroke 2008, 39:924-928.

3. Martin-Montalvo A, Mercken EM, Mitchell SJ, Palacios HH, Mote PL, Scheibye-Knudsen M, Gomes AP, Ward TM, Minor RK, Blouin MJ, Schwab M, Pollak M, Zhang Y, Yu Y, Becker KG, Bohr VA, Ingram DK, Sinclair DA, Wolf NS, Spindler SR, Bernier M, de Cabo R: Metformin improves healthspan and lifespan in mice. Nat Commun 2013, 4:2192.

4. Calvert JW, Gundewar S, Jha S, Greer JJ, Bestermann WH, Tian R, Lefer DJ: Acute metformin therapy confers cardioprotection against myocardial infarction via AMPK-eNOS-mediated signaling. Diabetes 2008, 57:696-705.

5. Group. UPDSU: Effect of intensive blood-glucose control with metformin on complications in overweight patients with type 2 diabetes (UKPDS 34). UK Prospective Diabetes Study (UKPDS) Group. Lancet 1998, 352:854-865.

6. Diamanti-Kandarakis E, Paterakis T, Alexandraki K, Piperi C, Aessopos A, Katsikis I, Katsilambros N, Kreatsas G, Panidis D: Indices of low-grade chronic inflammation in polycystic ovary syndrome and the beneficial effect of metformin. Hum Reprod 2006, 21:1426-1431.

7. Mihaylova MM, Shaw RJ: The AMPK signalling pathway coordinates cell growth, autophagy and metabolism. Nat Cell Biol 2011, 13:1016-1023.

8. Dasgupta B, Milbrandt J: Resveratrol stimulates AMP kinase activity in neurons. Proc Natl Acad Sci U S A 2007, 104:7217-7222.

9. Davis BJ, Xie Z, Viollet B, Zou MH: Activation of the AMP-activated kinase by antidiabetes drug metformin stimulates nitric oxide synthesis in vivo by promoting the association of heat shock protein 90 and endothelial nitric oxide synthase. Diabetes 2006, 55:496-505.

10. Sasaki H, Asanuma H, Fujita M, Takahama H, Wakeno M, Ito S, Ogai A, Asakura M, Kim J, Minamino T, Takashima S, Sanada S, Sugimachi M, Komamura K, Mochizuki N, Kitakaze M: Metformin prevents progression of heart failure in dogs: role of AMP-activated protein kinase. Circulation 2009, 119:2568-2577.

11. Spasic MR, Callaerts P, Norga KK: AMP-activated protein kinase (AMPK) molecular crossroad for metabolic control and survival of neurons. Neuroscientist 2009, 15:309-316.

12. Hattori Y, Suzuki K, Hattori S, Kasai K: Metformin inhibits cytokine-induced nuclear factor kappaB activation via AMP-activated protein kinase activation in vascular endothelial cells. Hypertension 2006, 47:1183-1188.

13. O'Neill LA, Hardie DG: Metabolism of inflammation limited by AMPK and pseudo-starvation. Nature 2013, 493:346-355.

14. Choi JS, Park J, Suk K, Moon C, Park YK, Han HS: Mild Hypothermia Attenuates Intercellular Adhesion Molecule-1 Induction via Activation of Extracellular Signal-Regulated Kinase-1/2 in a Focal Cerebral Ischemia Model. Stroke Res Treat 2011, 2011:846716.

15. Chopp M, Li Y, Jiang N, Zhang RL, Prostak J: Antibodies against adhesion molecules reduce apoptosis after transient middle cerebral artery occlusion in rat brain. J Cereb Blood Flow Metab 1996, 16:578-584.

16. Greenwood J, Mason JC: Statins and the vascular endothelial inflammatory response. Trends Immunol 2007, 28:88-98.

17. Strecker JK, Sevimli S, Schilling M, Klocke R, Nikol S, Schneider A, Schabitz WR: Effects of G-CSF treatment on neutrophil mobilization and neurological outcome after transient focal ischemia. Exp Neurol 2010, 222:108-113.

18. Yenari MA, Han HS: Neuroprotective mechanisms of hypothermia in brain ischaemia. Nat Rev Neurosci 2012, 13:267-278.

19. Wasserman JK, Schlichter LC: Minocycline protects the blood-brain barrier and reduces edema following intracerebral hemorrhage in the rat. Exp Neurol 2007, 207:227-237. 
20. Wang J, Gallagher D, DeVito LM, Cancino Gl, Tsui D, He L, Keller GM, Frankland PW, Kaplan DR, Miller FD: Metformin activates an atypical PKC-CBP pathway to promote neurogenesis and enhance spatial memory formation. Cell Stem Cell 2012, 11:23-35.

21. He X, Li Y, Lu H, Zhang Z, Wang Y, Yang GY: Netrin-1 overexpression promotes white matter repairing and remodeling after focal cerebral ischemia in mice. J Cereb Blood Flow Metab 2013, 33:1921-1927.

22. Liu Y, Tang GH, Sun YH, Lin XJ, Wei C, Yang GY, Liu JR: The protective role of Tongxinluo on blood-brain barrier after ischemia-reperfusion brain injury. J Ethnopharmacol 2013, 148:632-639.

23. Huang J, Li Y, Tang Y, Tang G, Yang GY, Wang Y: CXCR4 antagonist AMD3100 protects blood-brain barrier integrity and reduces inflammatory response after focal ischemia in mice. Stroke 2013, 44:190-197.

24. Takata F, Dohgu S, Matsumoto J, Machida T, Kaneshima S, Matsuo M, Sakaguchi S, Takeshige Y, Yamauchi A, Kataoka Y: Metformin induces up-regulation of blood-brain barrier functions by activating AMP-activated protein kinase in rat brain microvascular endothelial cells. Biochem Biophys Res Commun 2013, 433:586-590.

25. Force ICGT: Global Guideline for Type 2 Diabetes: recommendations for standard, comprehensive, and minimal care. Diabet Med 2006, 23:579-593.

26. Li J, Benashski SE, Venna VR, McCullough LD: Effects of metformin in experimental stroke. Stroke 2010, 41:2645-2652.

27. Harada S, Fujita-Hamabe W, Tokuyama S: The importance of regulation of blood glucose levels through activation of peripheral 5'-AMP-activated protein kinase on ischemic neuronal damage. Brain Res 2010, 1351:254-263.

28. Li W, Qu Z, Prakash R, Chung C, Ma H, Hoda MN, Fagan SC, Ergul A: Comparative analysis of the neurovascular injury and functional outcomes in experimental stroke models in diabetic Goto-Kakizaki rats. Brain Res 2013, 1541:106-114.

29. Gidday JM: Cerebral preconditioning and ischaemic tolerance. Nat Rev Neurosci 2006, 7:437-448.

30. Shibata R, Sato K, Pimentel DR, Takemura Y, Kihara S, Ohashi K, Funahashi T, Ouchi N, Walsh K: Adiponectin protects against myocardial ischemiareperfusion injury through AMPK- and COX-2-dependent mechanisms. Nat Med 2005, 11:1096-1103.

31. Nishimura M, Izumiya Y, Higuchi A, Shibata R, Qiu J, Kudo C, Shin HK, Moskowitz MA, Ouchi N: Adiponectin prevents cerebral ischemic injury through endothelial nitric oxide synthase dependent mechanisms. Circulation 2008, 117:216-223.

32. Salminen A, Kaarniranta K: AMP-activated protein kinase (AMPK) controls the aging process via an integrated signaling network. Ageing Res Rev 2012, 11:230-241.

33. Tak PP, Firestein GS: NF-kappaB: a key role in inflammatory diseases. J Clin Invest 2001, 107:7-11.

34. Isoda K, Young JL, Zirlik A, MacFarlane LA, Tsuboi N, Gerdes N, Schonbeck U, Libby P: Metformin inhibits proinflammatory responses and nuclear factor-kappaB in human vascular wall cells. Arterioscler Thromb Vasc Biol 2006, 26:611-617.

35. Chopp M, Zhang ZG, Jiang Q: Neurogenesis, angiogenesis, and MRI indices of functional recovery from stroke. Stroke 2007, 38:827-831.

36. Potts MB, Lim DA: An old drug for new ideas: metformin promotes adult neurogenesis and spatial memory formation. Cell Stem Cell 2012, 11:5-6.

doi:10.1186/s12974-014-0177-4

Cite this article as: Liu et al:: Metformin attenuates blood-brain barrier disruption in mice following middle cerebral artery occlusion. Journal of Neuroinflammation 2014 11:177.

\section{Submit your next manuscript to BioMed Central and take full advantage of:}

- Convenient online submission

- Thorough peer review

- No space constraints or color figure charges

- Immediate publication on acceptance

- Inclusion in PubMed, CAS, Scopus and Google Scholar

- Research which is freely available for redistribution 